\title{
Hernia Diafragmática Derecha en Lactante
}

\author{
Dr. Jorge Serpell B. ${ }^{1} ;$ Dra. Marisol Escobar M. ${ }^{2-}$
}

\section{RIGHT DLAPHRAGMATIC HERNLA IN INFANTS}

A case of right sided Bochdalek's hernia in an infant 1 ycars and 10 months old, in association with pulnonary sequestration is reported. Embriology, pathophysiology, diagnosis and treatment of the congenital diaphragmatic hernias are discussed, with emphasis in the persistently high mortality of symptomatic nconates, contrasting to the excellent prognosis of patients with delayed presentation.

La hernias diafragmáticas congénitas constitu. yen una patología muy importante en el periodo neonatal por su gravedad que condiciona una gran mortalidad y requiere un diagnóstico precoz $y$ un tratamiento medico quirurgico agresivo e in. tensivo. Se presentan con una frecuencia de $1 \times 1.500$ a $\mathrm{l} \times 1.800$ recién nacidos. Algunos no se diagnostican en el período neonatal, presentando sintomatologia más tardia, siendo habitualmente un hallazgo radiológico.

Las del lado derecho son raras representando de 10 a $150 \%$ del total, los sintomas tienden a ser más atenuados y el abordaje quirúrgico debe hacerse por vía torácica por la presencia subdiafragnática del hígado.

\section{Caso Clínico}

D.S.A., 2 arios de edad. Desde los cuatro meses de edad tenia sintomas bronquiales frecuentes y cansancio facil. A la edad de 1 ar̃o y 6 meses en una radiografía de tórax se demostró una imagen redondeada densa posterior en el hemitórax derecho que hacía cuerpo con el diafragma (Fig.1) y en la radioscopía presentaba movimientos oscilatorios con la respiración. Em. barazo y parto normales. Peso de nacimiento 3.450 g., Apgar 9 al minuto, sin otros antecedentes. En el examen físico de ingreso tenía palidez discreta de piel y mucosas, presión arterial $80 / 50$ $\mathrm{mm}$. de Hg., tórax simétrico, roncus bilaterales, matidez y disminución del murmullo vesicular en la base derecha. Exámenes de laboratorio y gases en sangre normales. Tránsito intestinal y enema baritada nomales. Fue intervenido a través de toracotomía posterolateral derecha, se comprobó una hernia de Bochdalek con saco pleuroperitoneal y hernia patcial del lóbulo hepático derecho; gran adherencia del segmento posterior del lóbulo inferior que se libera, $y$ vasos sanguineos anormales transdiafragmáticos que fueron ligados, concluyendo que se trataba de un secuestro pulmonar extralobar, que se extirpó. El saco hemiario fue plegado sin extirparlo y se

J Unidad de Cirugia Intantil. Hospital de lquique.

2 Servicio de Pediatria. Hospital de lquiejuc. cerró la brecha diafragmática con puntos en $(I$. Se dẹó drenaje pleural bajo agua e indicó ampicilina y kinesiterapia postoperatoria, presentando desde el tercer día sintomas bronquiales obstructivos que obligaron a usar cefradina, broncodilatadores y fluidificantes con evolución posterior favorable. La radiografía de control un mes después mostró sólo reacción pleural basal derecha. y tres meses después de operado estaba sin sintomas. (Fig. 2).

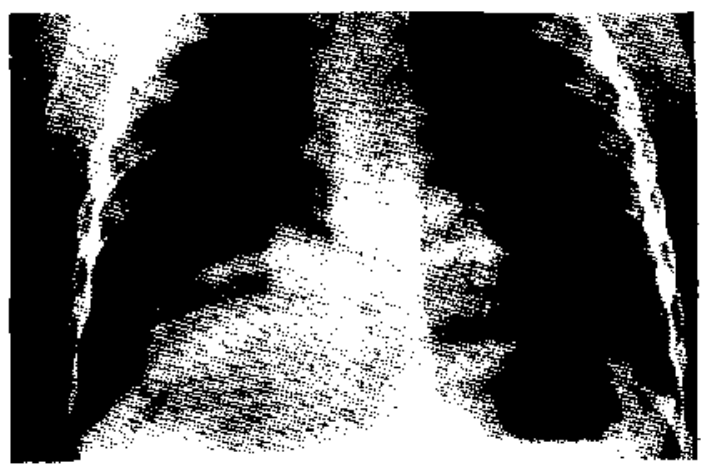

Figura 1: Kadiografía de Tórax mucstra imagen redondeada quc hace cuerpo con e] diaframa derecho.

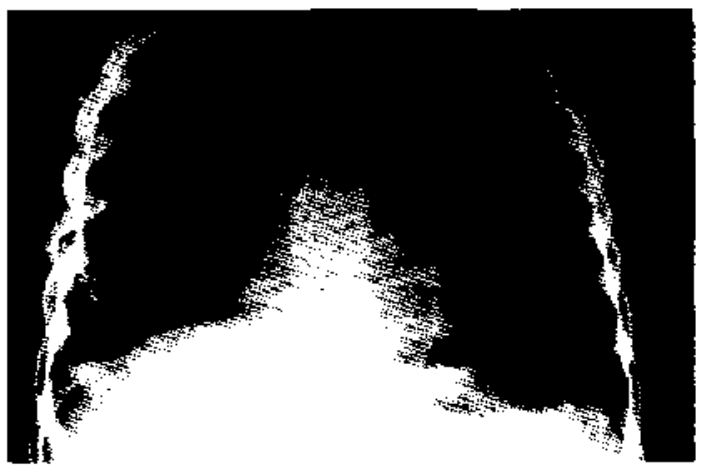

Figura 2: Radiogratía de contral 3 meses después de la intervención.

\section{COMENTARIO}

Las heridas de Bochdalek se presentan habitualmente en las primeras horas de vida con sin- 
tomas y signos respiratorios importantes $y$ gran mortalidad, representando un desafio terapéutico médico quirúrgico.

Ellas están constituídas por un defecto embrionario en el cierre del canal pleuroperitoneal en la parte posterior del diafragma, cuando el intestino primitivo regresa al abdomen, persistiendo el orificio posterior de Bochdalek, con persistencia de visceras abdominales en el tórax ${ }^{6-8}$ Estas profucen compresión del tejido pulmonar que, si ocurre antes de las 16 semanas de gestación lleva a una hipoplasia pulmonar con menor número de dirisiones bronquiales, ${ }^{2-4}$.

Si alcanzan a desarrollarse las hojas pleuroperitoneales $\sin$ formación del músculo, queda una hernia cubierta de saco, lo que es raro: En su gran mayoría no tienen saco. Si ocurre desarrollo de músculo, pero insuficiente, se producen eventraciones diafragmáticas.

Las hernias del lado derecho son bastantes raras, representan de 10 a $200 \%$ del total ${ }^{1-6-8}$, debido fundamentalmente a que el canal pleuroperitoneal derecho se cierra primero, son mejor toleradas se presentan habitualmente en forma tardía y el contenido está formado casi siempre por higado.

Las anomalías asociadas son poco frecuentes, con excepción de la male rotación intestinat. Se observan malformaciones cardíacas, gastrointestinales, genitourinarias y defectos de la pared abdominal en menos del $100 / 0$ de los pacjentes ${ }^{8}$. Se ha descrito la asociación con secuestro pulmonar, como ocurrió en este caso.

A pesar de los avances en el manejo de estos núnos, la mortalidad continúa siendo alta, especialmente en los casos con sintomas neonatales en que es mayor del $15 \%$. En cambio en los casos de presentación tardía es menor de $10 \%$. Se debe fundamentalmente, la mortalidad, a una insuficiencia respiratoria condicionada por hipoplasia pulmonar, debido a la comprensión por las visceras hemiadas ${ }^{2-4-6}$. Se producen hipoxia, hipercapnia $y$ acidosis que provocan hipertensión pulmonar progresiva, ésta lleva a corto circuito derecha izquierda a través del ductus y el forámen oval, y a mayor hipoxia, estableciéndose un círculo vicioso que conduce a la muerte de estos niños.

Algunos casos presentan escasos sintomas o no los tienen en el período neonatal, siendo diagnosticados habitualmente por hallazgos radiológicos posteriores. Los síntomas son respiratorios a digestivos, inespecíficos, aunque pueden ocurrir complicaciones digestivas agudas como oclusión intestinal o apendicitis herniarias ${ }^{\mathrm{i2}}$.

El tratamiento de las hernias de Bochdalek es quirúrgico. En los recién nacidos la intervención es perentoria con un manejo pre y postoperatorio enérgico. El abordaje es abdominal en las hernias izquierdas y por torácico en las derechas: se efectúa reducción de las vísceras a la cavidad abdominal y cierre de la brecha diafragmática previa instalación de un drenaje pleural. Habitualmente se puede efectuar el cierre del orificio sin problemas, aunque si éste es de gran tamafro puede ser necesario utilizar cierre mallas sintéticas a con colgajos musculares del músculo dorsal ancho ${ }^{3}$.

A diferencia de las formas neonatales, en que el pronóstico es malo y la mortalidad muy alta, en las de presentación tardía el pronóstico es excelente, con una sobrevida cercana al $1000 \%$.

Por último es importante mencionar el promisorio futuro de esta patología en base al diagnóstico prenatal y corrección de la hernia in utero, tratamiento que se halla en la etapa experimental ${ }^{9}$.

\section{RESUMEN}

Se presenta un caso de hernia de Bochdalek derecha en un lactante mayor, asociada con secuestro pulmonar. Se comenta la embriología, fisiopatología, diagnóstico y terapéutica de las hernias diafragmáticas, destacando la alta mortalidad que se mantiene en las formas neonatales en contraste con el excelente pronóstico en las formas de presentación tardía.

\section{REFERENCIAS}

${ }^{1}$ Arretz C.. Correia $G .$, Gomez $O$. et al: "Hernias y eventraciones diatragmáticas congénitas en el niño". Cuademo Chileno de Cirugía. 20: 84, 1976.

2 Bechroont T., Bondourn J.M. and Jaubert de Beaujeu M.: "The lungs in congenital diaphragnatic hernia". Chir. Pediatr. 19: 145, 1978

${ }^{3}$ Brandesky' $6:$ "Results with the rives-plasty in the treatment of congenital diaphragmatic defects and eventration", Z. Kindenchir. 21: 123, 1979.

4 Dibbins, A. W.: "Hernja diagramática congénita, pulmón hipoplástico y vasoconstricción pulmonar" Clin. Perinatol. 5: 93, 1978.

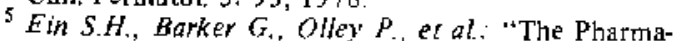
cologic treatment of newborn diaphragnatic hernia A. 2-year evaluation". Pediatr. Surg. 15: 384, 1980.

${ }^{6}$ Erlich F.E., Soirberg A.M.: "Pathophysiology and management of congenital posterolateral diaphragmatic hernias". An. Surg. 44: 26, I9?8.

${ }^{7}$ Golladay E.S., Katz J.R., Katz H, et al: "Delayed presentation of congenital postcrolateral diaphragmatic hernia". A dramatic catse of faihre to thrive Pediats. Surg. 16: 503, 1981.

B Harrison M.R. and DE Lorimier A.A.: "Congenital diaphragmatic hernia". Surg. Clin. North Am 61: $1023,1981$.

${ }^{9}$ Harrison M.R., Ross $N$.A. and De Lormier A.A.: "Corrcetion of congenital diaphragunatic hernja in 
utero II development of a succesful surgical tech. nique using abdominoplasty to avoid compromisc of umbilical blood flow". Pediatt. Surg. 16; 934 , 1981.

10 Kerr A.A.: "Lung function in children after repair of congenital diaphragmatic hernia". Arch. Dis.
Child. 52: 902, 1977 .

11 Ruff S.J., Campbell $J . R$., Harrison M. W' et at.: "Pediatric diaphragmatic hernia an 11 year experience". Am. Surg. 139, 1980.

12 Saffie A.: "Hernia de Bochdalek en un precscolar". Rev. Clil. Pediat. 52: 233, 1981 